\title{
Descripción del comportamiento de películas plásticas multicapa en procesos de termodeformación mediante análisis de fotoelasticidad digital
}

\author{
$\checkmark$ FERnANDo MELÉndEZ LOZADA ${ }^{1}$ \\ Alejandro Restrepo MartíneZ ${ }^{2}$ \\ FRANCISCO EUGENIO LÓPEZ GIRALDO ${ }^{1}$
}

\section{Resumen}

El objetivo de este artículo es describir en términos del espesor el comportamiento dinámico de la película plástica multicapa en un proceso de termodeformación bajo dos escenarios de temperatura $\left(\mathrm{T}_{1}=20^{\circ} \mathrm{C}, \mathrm{T}_{2}=60^{\circ} \mathrm{C}\right)$ usando la fotoelasticidad como técnica de no contacto. Los colores de interferencia presentados en las imágenes de fotoelasticidad están asociados al espesor de la película plástica y a través del entrenamiento de un algoritmo de aprendizaje tipo ANFIS se relaciona color con espesor. La red neuro-difusa asocia cada intensidad de color del pixel con un espesor medido, obteniendo a la salida una imagen de espesores en escala de grises. Se concluye que los colores de interferencia están directamente correlacionados con el espesor de la película y la temperatura favorece la deformación permitiendo llegar a espesores menores en menor tiempo.

Palabras clave: Fotoelasticidad digital, Anfis, Aprendizaje de color, Visión artificial.

\section{Description of Multilayer Plastic Films Behavior in Thermodeformation Processes by Digital Photoelasticity Analysis}

\section{Abstract}

The aim of this paper is describes in thickness terms the dynamic behavior of the multilayer plastic film in a thermoforming process under two temperature conditions $\left(\mathrm{T} 1=20^{\circ} \mathrm{C}, \mathrm{T} 2=60^{\circ} \mathrm{C}\right)$ using the photoelasticity how no-contact

1 Instituto Tecnológico Metropolitano - ITM, Medellín, Colombia.

2 Universidad Nacional de Colombia, sede Medellín, Colombia.

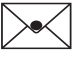

Autor de correspondencia: Meléndez Lozada, F. (Fernando): Carrera 26H 52-46, Cali, Colombia. Teléfono: 3166323179. Correo electrónico: fher.melendez@gmail.com
Historia del artículo:

Artículo recibido: 4-IX-2017 / Aprobado: 30-V-2018

Disponible online: 3 de agosto 2018

Discusión abierta hasta abril de 2020 
technique. The interference colors showed on the photoelastic images is associated with the thickness of the plastic film and through the training of a learning algorithm type ANFIS is related color with thickness. The neuro-fuzzy net associated to each color intensity of pixel with the thickness measured, obtaining at the output a thickness image in gray scale. It is concluded that interference colors are directly correlated with the film thickness and the temperature favors the deformation allowing to achieve in smaller thicknesses in less time.

Keywords: Digital photoelasticity, Anfis, Color learning, Artificial vision.

\section{Descrição do comportamento de filmes plásticos multicamada em processos de termo deformação por análise de fotoelasticidade digital}

\section{Resumo}

O objetivo deste artigo é descrever em termos de espessura o comportamento dinâmico da película plástica multicamada em um processo de termoformação em dois cenários de temperatura $\left(\mathrm{T} 1=20^{\circ} \mathrm{C}\right.$, $\left.\mathrm{T} 2=60^{\circ} \mathrm{C}\right)$ usando fotoelasticidade como técnica sem contato. As cores de interferência apresentadas nas imagens de fotoelasticidade estão associadas à espessura da película plástica e através do treinamento de um algoritmo de aprendizado tipo ANFIS é cor relacionada com a espessura. A rede neuro-difusa associa cada intensidade de cor do pixel com uma espessura medida, obtendo na saída uma imagem de espessuras em escala de cinza. Concluise que as cores de interferência estão diretamente correlacionadas com a espessura do filme e a temperatura favorece a deformação, permitindo atingir espessuras menores em um curto período de tempo.

Palavras-chave: Fotelasticidade digital, Anfis, Aprendizagem de cores, Visão artificial.

\section{Introducción}

En la industria se emplean películas plásticas multicapa (PPMC) como materia prima para el empacado, estas brindan una barrera hermética que ayuda a la preservación e inocuidad de los productos a conservar. Las PPMC son sometidas a procesos industriales de moldeo por termodeformación en donde sufren cambios en la capa de barrera que evita el intercambio de gases; esto se presenta por la reducción del espesor debido al estiramiento y concentración de esfuerzos en el área de mayor deformación, además la temperatura afecta la homogeneidad del espesor siendo este un indicador importante de calidad para la industria de alimentos (Agudelo, 2009). Por otra parte la inspección de calidad del empaque termoformado se realiza de ma- nera puntual e invasiva lo cual representa pérdidas no solo en materia prima sino también en tiempo de procesamiento; por esta razón es necesario considerar la estimación del espesor en áreas de mayor concentración de esfuerzos bajo una técnica de medición de no contacto con el fin de describir su comportamiento durante la deformación.

El material de las PPMC son plásticos traslucidos y ópticamente isotrópicos cuando están libres de esfuerzo mecánico, pero al ser sometidos a carga mecánica (tensión) se transforman en materiales anisotrópicos, los cuales presentan birrefringencia. Esta característica óptica permite el análisis por fotoelasticidad digital, la cual es una técnica experimental de campo completo que consiste en la adquisición de imágenes a color del fenómeno fotoelástico 
visualizado por la utilización de un montaje óptico de polariscopio plano que consiste en hacer pasar luz blanca polarizada a través de la PPMC y capturar el video de la deformación con una cámara RGB anteponiendo un analizador lineal sobre el lente. Las imágenes adquiridas muestran franjas de color, las cuales proveen la información de la diferencia de los esfuerzos principales que están asociados al retardo de fase, espesor y coeficiente de esfuerzo óptico del material (Ashokan \& Ramesh, 2009; Briñez et al. 2012). Adicionalmente, el espesor juega un papel relevante en la descripción del comportamiento de la PPMC sometidas a termodeformación ya que éste afecta las capacidades funcionales de las películas como barrera protectora.

Las técnicas más comunes de obtención de información fotoelástica para materiales plásticos anisotrópicos son el método de cambio de fase "phase shifting", análisis del contenido espectral "spectral content analysis", transformada rápida de Fourier y fotoelasticidad RGB entre otros (Ajovalasit et al. 2015; Swain et al. 2015); estas buscan determinar las zonas de mayor esfuerzo, parámetros isoclínicos y el retardo de fase, para una imagen sobre un espécimen cargado estáticamente.

Para la estrategia de phase shifting se deben adquirir al menos cuatro imágenes simultáneas con configuraciones ópticas diferentes (Ramesh \& Ramakrishnan, 2011). Con estas se obtiene una imagen de fase envuelta y que requiere técnicas de desenvolvimiento de fase. Así que phase shifting tiene como desventaja la complejidad del montaje y susceptibilidad de la calibración de los elementos ópticos empleados.

En el caso del spectral content analysis se usa para estimar el retardo de fase y el número de patrones de intensidad, en este se realiza mediciones de manera puntual bajo un rango de longitudes de onda usando un espectrofotómetro, los valores de intensidad en cada punto medido sobre la franja se compara con los valores teóricos de intensidades correspondientes al rango apropiado de retardo (SolagurenBeascoa Fernández, 2011; Kulkarni \& Rastogi, 2016).
En cuanto a la fotoelasticidad RGB es un método de campo completo que determina el orden de la franja y su proceso inicial es generar una tabla de consulta de color calibrado (LUT-"Look-up Table”) versus el desfase, luego con la imágenes obtenidas del modelo el color es demodulado por comparación contra la LUT usando una función de minimización del error (Swain, Philip, \& Pillai, 2014); Este método tiene como dificultad la obtención de una LUT que se aproxime con precisión a los colores de interferencia que se presentan en las imágenes de resultado, la ventaja de esta es el no requerimiento de las propiedades ópticas del material (Swain et al., 2015).

A pesar de la amplia investigación que se ha llevado a cabo sobre fotoelasticidad, existen pocos estudios sobre el análisis de espesores a partir de imágenes de fotoelasticidad y más aun con cargas dinámicas de deformación en películas plásticas multicapa. Algunos trabajos recientes han demostrado la existencia de la relación entre la deformación con los colores de interferencia. Briñez et al. (2013b) emplean la fotoelasticidad y métricas de similitud a través de correlación digital de imágenes para la caracterización del comportamiento local de los patrones temporales de franjas de color en la película plástica durante la deformación mecánica a temperatura ambiente, arrojando como resultado la diferenciación entre las zonas de comportamiento elástico y viscoelástico. En el mismo año Briñez et al. (2013a) muestran el análisis de la evolución de intensidades RGB de los pixeles en puntos fijos de observación que evidencian que la PPMC presentan comportamientos ópticos no homogéneos sobre toda la superficie. Utilizando análisis morfológicos los autores Briñez et al. (2013c) a través de las técnicas de análisis de imágenes como estereología y superficies erosionadas determinaron el crecimiento gradual del tamaño de las franjas de color y encontraron una relación de las franjas con el comportamiento mecánico durante el proceso de deformación permitiendo así distinguir la zona elástica y viscoelástica del material. Dos años después se propone utilizar descriptores espacio-temporales de los patrones de las franjas de 
color tomando los cambios de orden como una variable asociada a la distribución de esfuerzos, realizando la segmentación del tono magenta y análisis de cambios de saturación demostrando que a mayores cambios de orden aumenta la saturación (Briñez, Restrepo, \& Branch, 2015). En general se encuentra que los estudios no incluyen la temperatura como una variable adicional al proceso de deformación.

Recientemente hemos desarrollado varias aproximaciones para valorar la influencia de la temperatura en las propiedades de las imágenes de fotoelasticidad. En el trabajo de Meléndez et al. (2015), se encontró que en la PPMC con adición de temperatura se requirió menor energía mecánica para su deformación, y se hicieron correlaciones de imágenes generadas a una temperatura de $60^{\circ} \mathrm{C}$. Sin embargo, estas correlaciones no están teniendo en cuenta el orden de las franjas ni el espesor de la PPMC. También se ha adentrado al campo del análisis de resistencia a la fractura de películas poliméricas a través de la combinación de pruebas de tracción y fotoelasticidad que permite seguir la geometría y la cantidad de deformación plástica siendo estos parámetros claves para la selección de las películas (Dubelley, Planes, Bas, Yrieix, \& Flandin, 2016).

Dado que la fotoelasticidad digital entrega información importante para la descripción del comportamiento de las PPMC durante la deformación, y las investigaciones han avanzado en determinar el retardo de fase y los esfuerzos principales a través de los colores de interferencia, pero el coeficiente óptico del material de la película multicapa es desconocido, por tal razón se tiene la necesidad de desarrollar la evaluación de la influencia en los procesos de termodeformación usando fotoelasticidad inversa valorando la posibilidad de usar la visión artificial como herramienta descriptiva de dichos ensayos.

Debido a la cantidad de procesos que se requieren para el análisis de imágenes de fotoelasticidad con estrategias convencionales y más aun con cambios dinámicos, se requiere el uso de algoritmos inteligentes en el estudio de las imágenes de fotoelasticidad. Los autores Grewal \& Dubey (2007), presentan una técnica de análisis inverso de franjas fotoelásticas utilizando redes neuronales para determinar la carga aplicada sobre un espécimen sometido a flexión, para ello utiliza información de las imágenes de las franjas fotoelásticas entregando como entrada para la red neuronal la intensidad del canal R y la salida es la deflexión del modelo. Este último parámetro es utilizado por la facilidad de medir durante el entrenamiento y está relacionado con la carga aplicada. La novedad de esta técnica es que la red neuronal es entrenada con datos de imagen directa de experimentos reales y no requiere cálculos previos ni conocimiento de fotoelasticidad.

En películas plásticas, Briñez et al. (2016), realizaron un trabajo que permite modelar como serie de tiempo la intensidad de cada pixel de la imagen durante la deformación usando redes neuronales recurrentes con el fin de identificar las zonas de mayor esfuerzo.

En este artículo se empleará un sistema neurodifuso para desarrollar un aprendizaje que permite relacionar colores de fotoelasticidad con espesor y a partir de esta herramienta se evaluarán las diferencias en los procesos de deformación en las películas cuando éstas son sometidas a dos temperaturas y demostrando de manera cualitativa como afecta la temperatura la deformación de la película plástica.

\section{Materiales y método}

El método planteado es experimental y de análisis cualitativo. En la Figura 1 se puede apreciar la metodología empleada y sus procesos experimentales la cual está dividida en cuatro fases.

\subsection{Termodeformación y adquisición} de ilmágenes

En esta fase 1 se adquieren las imágenes de fotoelasticidad para los dos escenarios de temperatura. Además las imágenes son recortadas en el área de interés para el análisis, lo que permite la generación correcta de datos relacionados al área que será analizada. 
Figura 1. Diagrama de la metodología y procesos experimentales

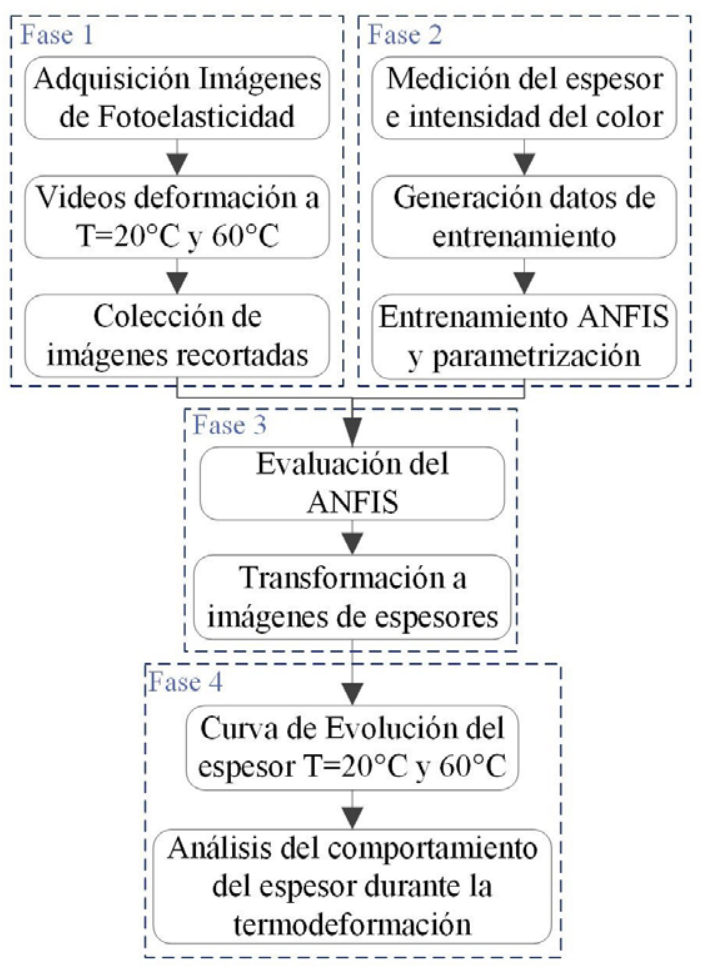

Para llevar a cabo el experimento es necesario implementar un montaje óptico de polariscopio pla- no, el cual consta de una fuente de iluminación que irradia a dos polarizadores lineales dispuestos en su línea de transmisión. En medio de los polarizadores se posiciona la muestra en evaluación que para nuestro caso será la película multicapa (PPMC). Se utiliza una configuración cruzada de los polarizadores, lo que corresponde a que los polarizadores se ubican perpendiculares entre sí, como se observa en la Figura 2. En este caso, la luz de la fuente de iluminación pasa a través del primer polarizador y emerge oscilando en un mismo plano (polarizada en el eje de transmisión del polarizador). Cuando el rayo de luz polarizada viaja a través de la muestra en estudio, se generan dos rayos ortogonales con un desfase $\delta$ entre ellos, y proporcional a los esfuerzos en el punto de evaluación. Finalmente estos rayos son alineados nuevamente por el segundo polarizador, el cual muestra la magnitud del retardo en términos de variaciones de intensidad. De este modo, la intensidad resultante de luz es una función del retardo $\delta$ y el ángulo entre el analizador y los esfuerzos principales $(\beta-\alpha)$. La intensidad de la luz emergente del polariscopio plano se aprecia en la Ecuación 1 (Navarro, Martínez, Saavedra, \& Pons, 2013):

\section{Figura 2. Polariscopio plano.}

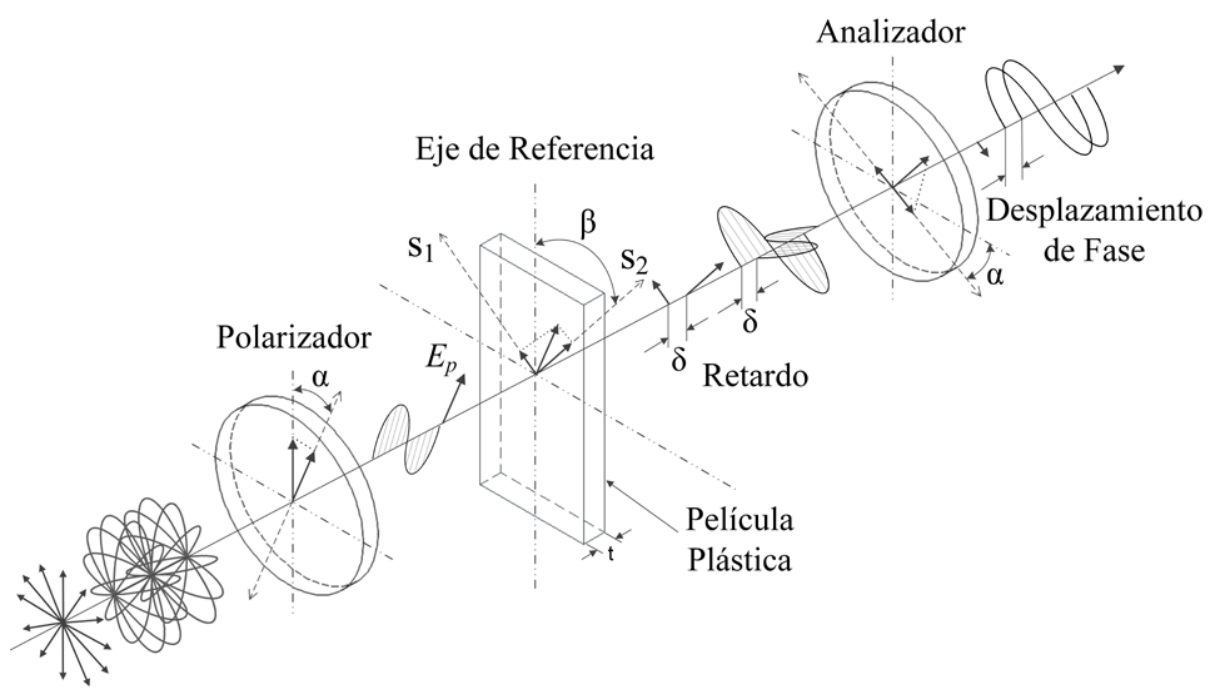

Rayos de Luz 


$$
I=E_{p}^{2} \operatorname{sen}^{2} 2(\beta-\alpha) \operatorname{sen}^{2}\left(\frac{\pi \delta}{\lambda}\right)
$$

Donde $E_{p}$ es la amplitud de la componente del campo eléctrico transmitido por el primer polarizador, $\lambda$ es la longitud de onda emitida por la fuente de luz, $\beta$ es el ángulo entre el esfuerzo principal $S_{2} y$ el eje de referencia y $\alpha$ es el ángulo del polarizador.

El montaje completo del experimento de termodeformación y fotoelasticidad se puede ver de manera simplificada en la Figura 3, en donde interviene una cámara RGB Edmund optics (ref: FL3-U388S2C-C), una lente (ref: K58-000), fuente de luz LCD Viewsonic (ref: VA1940w) y polarizadores lineales. Para el proceso de termodeformación se tiene una máquina de tracción Labthink (ref: Param XLW), un soplador de calor y una cámara termográfica FLIR (ref: AX5).

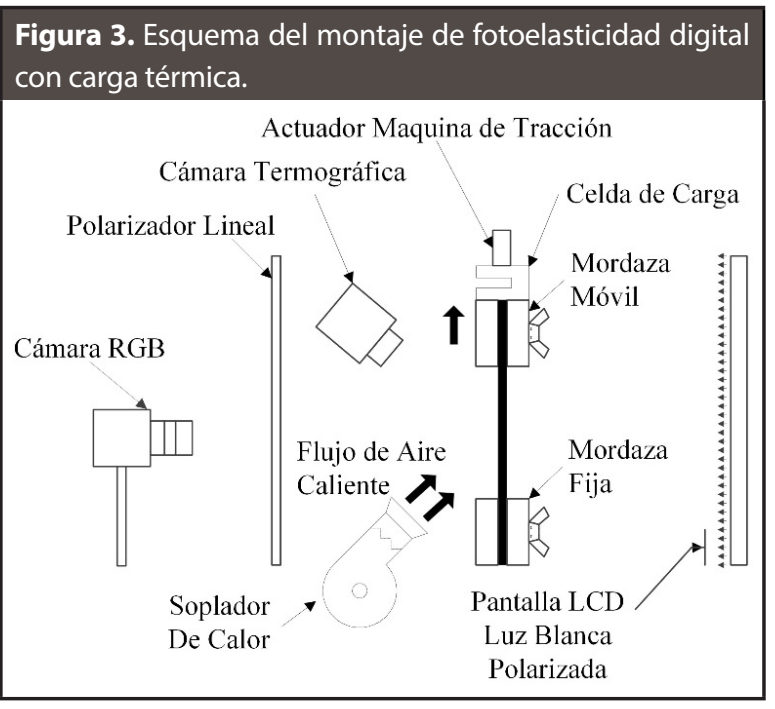

La preparación de la película plástica multicapa está basada en el estándar ASTM D882 (ASTM D882-02, 2002). Se puede observar la Figura 4 en donde las dimensiones de la PPMC son de ancho $=25 \mathrm{~mm}$, largo $=160 \mathrm{~mm}$ y $130 \mu \mathrm{m}$ de espesor. También el corte del material debe seguir al sentido longitudinal de las líneas de extrusión que la película plástica presenta.

La máquina de tracción es puesta en modo "ruptura y elongación" y se parametriza las dimensiones de la película plástica a deformar: longitud= $100 \mathrm{~mm}$ (separación libre entre mordazas), ancho= $25 \mathrm{~mm}$, espesor de la película $=0,130 \mathrm{~mm}$, velocidad de mordaza móvil $=500 \mathrm{~mm} / \mathrm{min}$. Luego de tener el montaje y las probetas, se procede a sujetar la PPMC en las mordazas de la máquina de tracción se enciende la fuente de luz, la cual será una pantalla LCD. El polarizador se gira hasta tener anulación de la luz $(\pi / 2$ respecto a la dirección de polarización de la pantalla); se habilita la aplicación de la cámara en modo video (30fps).

Para la deformación a temperatura ambiente se procede a iniciar la grabación del video e iniciar el experimento presionando el botón "test" de la máquina. En el caso de deformación de la película sometida a una temperatura superior a la ambiental, se enciende previamente al inicio el soplador de calor y a través de la cámara termográfica se verifica que la temperatura deseada en la PPMC sea cualitativamente homogénea en el área de interés (ROIRegion of interest).

Figura 4. Dimensiones de la PPMC.

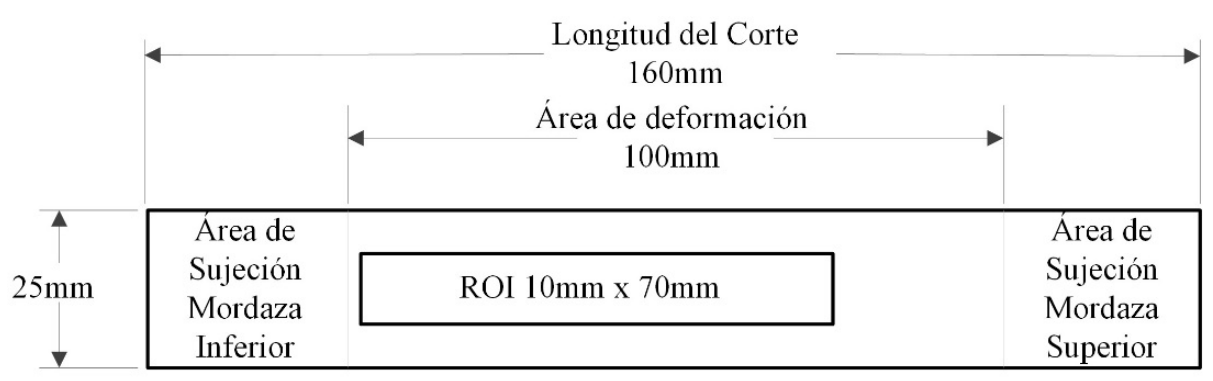


Se adquirieron 10 videos de deformación a temperatura ambiente $\mathrm{T}=20^{\circ} \mathrm{C}$ y 10 videos con carga térmica a $\mathrm{T}=60^{\circ} \mathrm{C}$, estos tienen una duración de aproximadamente 40 segundos a una tasa de $30 \mathrm{fps}$ (frames per second), posterior a la adquisición, por video 1200 imágenes son procesadas realizándole un recorte como se ve en la Figura 4 (dimensiones del recorte 1260x180 pixeles). El área de interés ROI ha sido ubicado en cercanía de la mordaza fija ya que en esta área se presenta mayor dinámica de la franjas de color.

En la Tabla 1 se muestran las imágenes más representativas del video tanto para $\mathrm{T}=20^{\circ} \mathrm{C}$ como $\mathrm{T}=60^{\circ} \mathrm{C}$. Las películas plásticas multicapa sometidas a termodeformación generan esfuerzos mecánicos que alteran las propiedades ópticas y mecánicas del material.

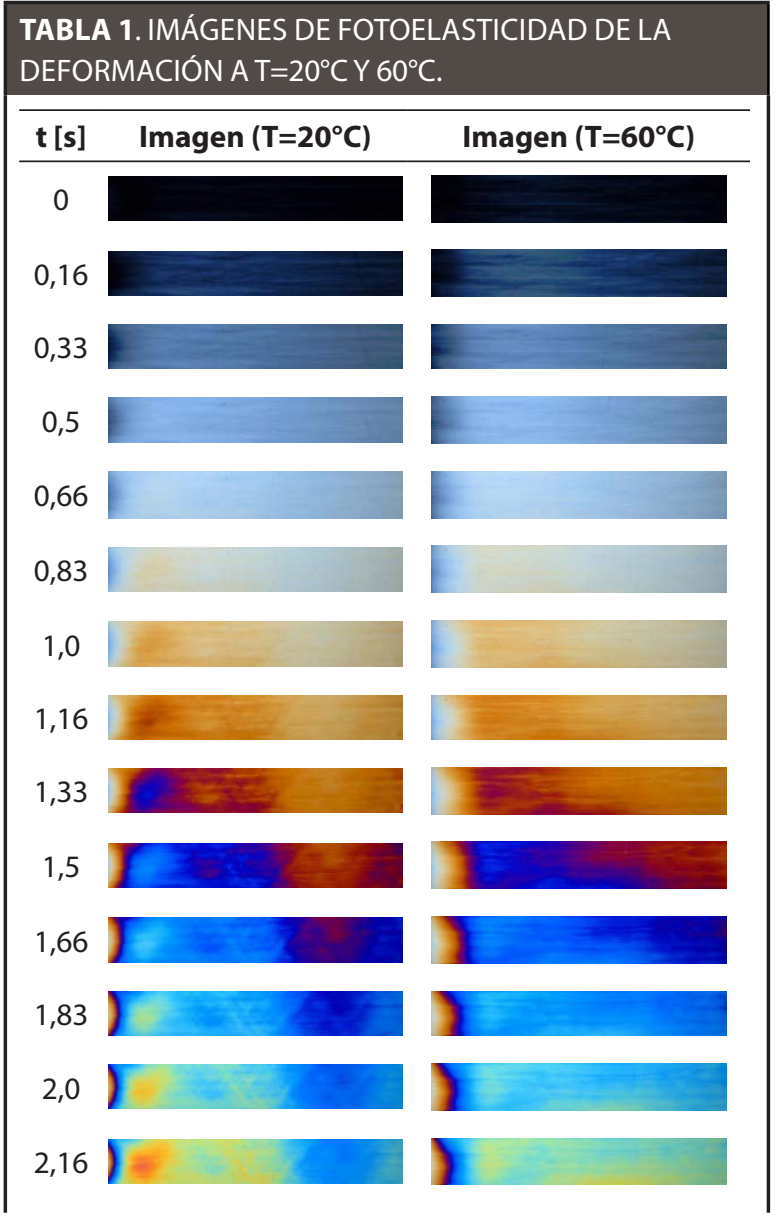

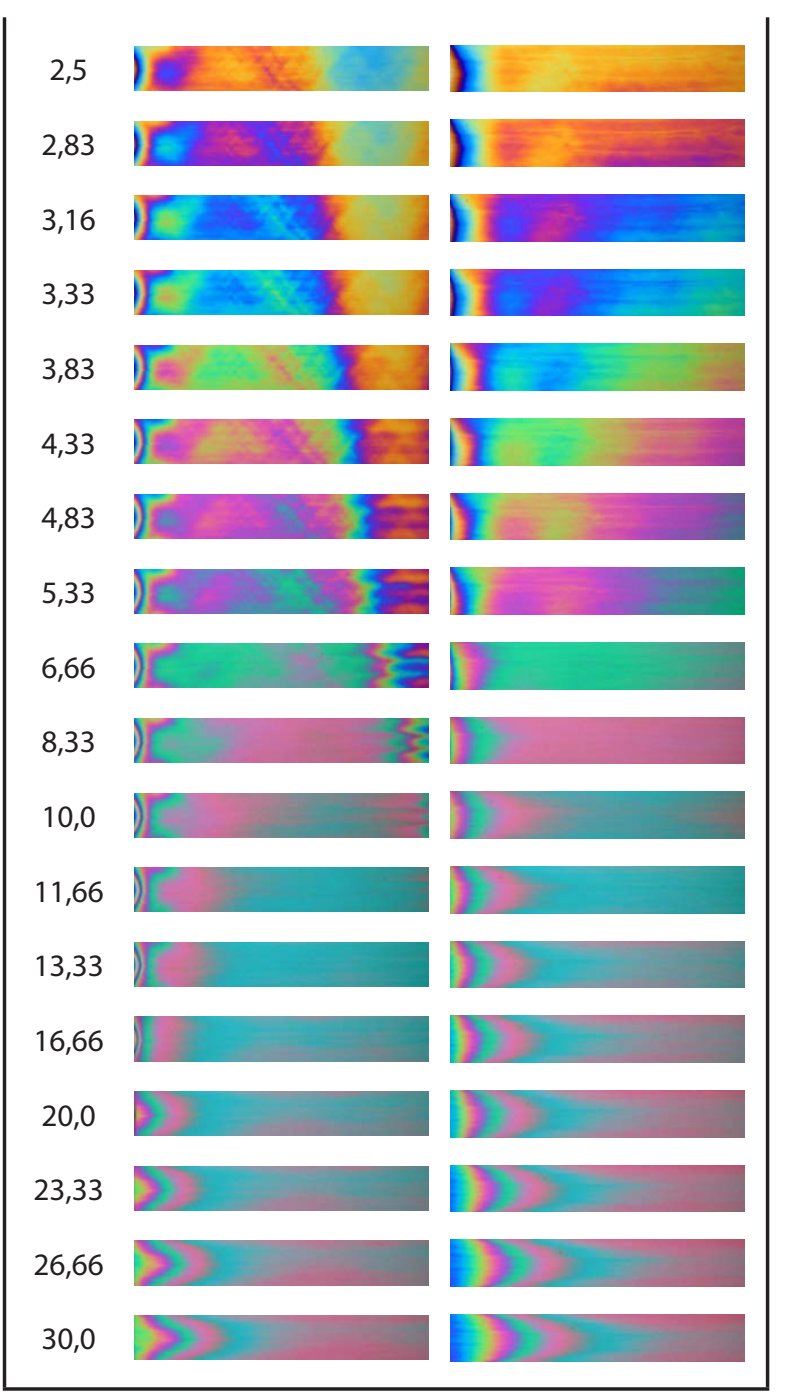

\subsection{Fase 2: Entrenamiento del ANFIS}

El espesor de la PPMC es un descriptor del comportamiento de la termodeformación debido a la relación entre los colores de interferencia y espesor, por lo tanto se plantea el uso de un sistema de inferencia neuro-difusa adaptativa (ANFIS- adaptive neuro fuzzy inference system) (Mitra \& Hayashi, 2000) que permite el aprendizaje del color-espesor de manera supervisada, empleando una base de datos de entrenamiento en donde la entrada son las intensidades de los canales de los modelos de color RGB y HSI y en la salida de la red se incorporan los datos de espesores medidos manualmente por un micrómetro con una resolución de 2.54 micras. 
Figura 5. Posición del recuadro de medición.

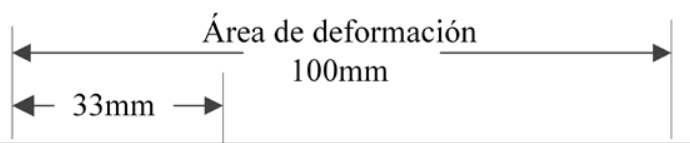

\begin{tabular}{|c|cc|}
\hline Área de & $\begin{array}{c}\text { Recuadro de } \\
\text { medición }\end{array}$ & $\begin{array}{c}\text { Área de } \\
\text { Sujeción } \\
\text { Sujeción } \\
\text { Mordaza } \\
\text { Inferior }\end{array} \quad \begin{array}{c}\text { Mordaza } \\
\text { Superior }\end{array}$ \\
\hline
\end{tabular}

Para la base de datos de entrenamiento se toman nueve mediciones puntuales dentro del recuadro marcado en la PPMC (ver Figura 5) en diferentes instantes de la deformación a $\mathrm{T}=20^{\circ} \mathrm{C}$. La máquina de tracción realiza 20 paradas cada 1,5s y en cada parada se adquiere la imagen de fotoelasticidad (ver Tabla 2) y se realiza las nueve mediciones de espesor con el micrómetro como se representa en la Figura 6, en las imágenes de la Tabla 2 la región de recuadro analizada se deforma debido al estiramiento de la película.

\section{TABLA 2. IMÁGENES RELACIONADAS A LA RESPECTIVA PARADA.}

\begin{tabular}{ccc} 
Parada & Imagen Adquirida & Recuadro \\
\hline 10 & \\
12 & \\
16 &
\end{tabular}

Figura 6. Puntos de medición dentro del recuadro.

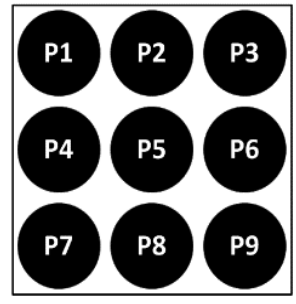

A través de un algoritmo se registran ordenadamente para cada punto ( $\mathrm{P} 1$ al $\mathrm{P} 9$ ) el promedio de las intensidades de los canales R, G, B, H, S e I (denotados IR, IG, IB, IH, IS e II) dichos datos serán las entradas de las red (ANFIS) y los datos de las salidas asignadas serán los valores de espesor (micras) medidos manualmente en cada una de las nueve posiciones (ver Tabla 3). Para el proceso de aprendizaje se generaron 1035 datos de entrenamiento. En la Tabla 4 se ejemplifican algunos casos del color analizado y el espesor medido en uno de los puntos.

\section{TABLA 3. ORGANIZACIÓN DE DATOS DE} ENTRENAMIENTO.

\begin{tabular}{c|c|c|c|c|c|c}
$\mathbf{e}$ & $\mathbf{R}$ & $\mathbf{G}$ & $\mathbf{B}$ & $\mathbf{H}$ & $\mathbf{S}$ & I \\
\hline $\mathrm{e}_{1}$ & IR P1 & IG P1 & IB P1 & IH P1 & IS P1 & II P1 \\
\hline $\mathrm{e}_{2}$ & IR P2 & IG P2 & IB P2 & IH P2 & IS P2 & II P2 \\
\hline$\ldots$ & $\ldots$ & $\ldots$ & $\ldots$ & $\ldots$ & $\ldots$ & $\ldots$ \\
\hline $\mathrm{e}_{1035}$ & IR P9 & IG P9 & IB P9 & IH P9 & IS P9 & II P9 \\
\hline
\end{tabular}

TABLA 4. COLOR-ESPESOR MEDIDO.

\begin{tabular}{cccc}
\hline Puntos & Medición & Puntos & Medición \\
\hline $134,9 \mu \mathrm{m}$ & $117,2 \mu \mathrm{m}$ \\
$133,7 \mu \mathrm{m}$ & $115,9 \mu \mathrm{m}$ \\
$132,4 \mu \mathrm{m}$ & $114,6 \mu \mathrm{m}$ \\
& $131,1 \mu \mathrm{m}$ & $109,6 \mu \mathrm{m}$ \\
$129,9 \mu \mathrm{m}$ & $100,7 \mu \mathrm{m}$ \\
$123,5 \mu \mathrm{m}$ & $98,10 \mu \mathrm{m}$ \\
\hline $119,7 \mu \mathrm{m}$ & $81,60 \mu \mathrm{m}$ \\
\hline
\end{tabular}


Con el conjunto de datos de entrenamiento generado se evalúan diferentes configuraciones de parámetros del ANFIS-Matlab® (ver Tabla 5) usando solo tres entradas (RGB) y se selecciona el mejor rendimiento, en este caso la función de pertenencia gaussiana combinada arrojo el menor error de $8.2 \%$. Con la función seleccionada se usa en el entrenamiento con seis entradas (RGBHSI) y tres funciones de pertenencia y 729 reglas basado en el modelo difuso Sugeno (Araya \& Cipriano, 2007) dando como resultado un error de $4.63 \%$ como se puede apreciar en la última fila de la Tabla 5. De lo anterior se puede concluir que para los datos analizados el HSI aporta en el proceso de descripción para mejorar la regresión respecto al espesor.

\section{TABLA 5. SELECCIÓN DE PARÁMETROS DEL ANFIS.}

\begin{tabular}{|c|c|c|c|}
\hline $\begin{array}{l}\text { Tipo de Función de } \\
\text { Pertenencia }\end{array}$ & $\begin{array}{l}\text { Épocas al } \\
\text { Mínimo } \\
\text { Error }\end{array}$ & Error & $\begin{array}{c}\% \\
\text { Error }\end{array}$ \\
\hline Triangular & 22 & 6,17 & 9,79 \\
\hline Trapezoidal & 36 & 6,28 & 9,96 \\
\hline Campana & 46 & 5,46 & 8,66 \\
\hline Gaussiana & 27 & 5,64 & 8,95 \\
\hline Gaussiana combinada & 70 & 5,17 & 8,20 \\
\hline Forma pi $(\pi)$ & 91 & 5,35 & 8,49 \\
\hline $\begin{array}{l}\text { Diferencia dos } \\
\text { sigmoidales }\end{array}$ & 70 & 5,34 & 8,47 \\
\hline $\begin{array}{l}\text { Producto dos } \\
\text { sigmoidales }\end{array}$ & 70 & 5,34 & 8,47 \\
\hline $\begin{array}{l}\text { Gaussiana combinada } \\
\text { (6 entradas) }\end{array}$ & 20 & 2,92 & 4,63 \\
\hline
\end{tabular}

El entrenamiento arroja una curva del error mostrado en la Figura 7 que se vuelve asintótica en el valor de error de 2,92 a partir de la época 20. El algoritmo de entrenamiento usado es una combinación de los métodos mínimos cuadrados y retropropagación con gradiente descendiente.

En la Figura 8 se muestra la salida con respecto a la cantidad de datos de entrenamiento (color azul), se puede apreciar que una línea con comportamiento lineal ya que los datos se ordenaron de manera ascendente. Luego del aprendizaje se evalúa el modelo
ANFIS dando como resultado los puntos rojos, en la cual hay dispersión en la zona de menor espesor y está asociada a que el número de orden de los colores de interferencia son altos y los colores cada vez son más similares, a pesar de la imprecisión de este método de "no contacto" permite aproximar el comportamiento de las PPMC e identificar diferencias entre los dos escenarios de temperaturas.
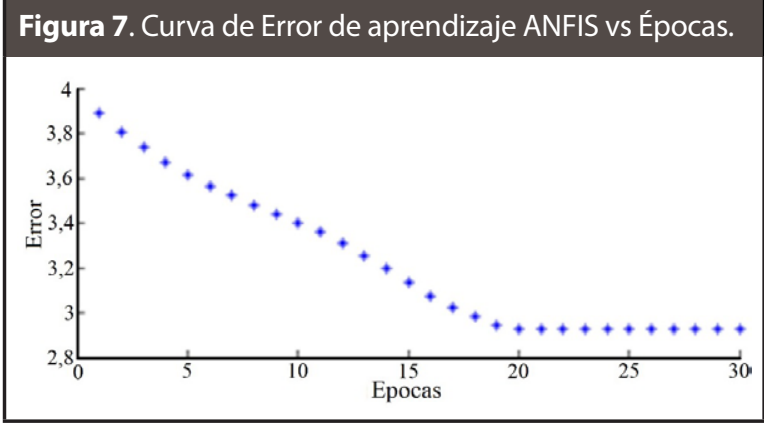

\section{Figura 8. Prueba de aprendizaje.}

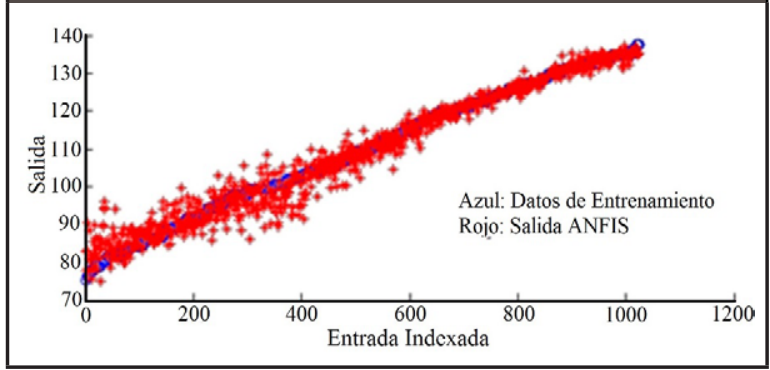

\section{Resultados}

En esta fase 3 con el ANFIS entrenado se evalúan las imágenes de fotoelasticidad y a su salida arroja un valor del espesor por cada pixel entre $0 \mathrm{y}$ 255 generando así una imagen en escala de grises que representa el espesor de 138 micras el color negro y a 75 micras el color blanco, la escala de gris es empleada para facilitar la inspección visual. Las imágenes de espesor muestran que la deformación es por regiones (ver Tabla 6 y 7) y las franjas se desplazan a medida que el material es estirado, algunos bordes de las franjas mantienen el espesor y también se puede observar diferencias en el comportamiento del espesor en la deformación a $\mathrm{T}=60^{\circ} \mathrm{C}$, en la Tabla 7 las imágenes logran valores de menor espesor (color 
blanco) para los cuadro 400, 600 y 800 , otra observación importante es en la homogeneidad del espesor en la zona de calentamiento cuando la PPMC es termodeformada.

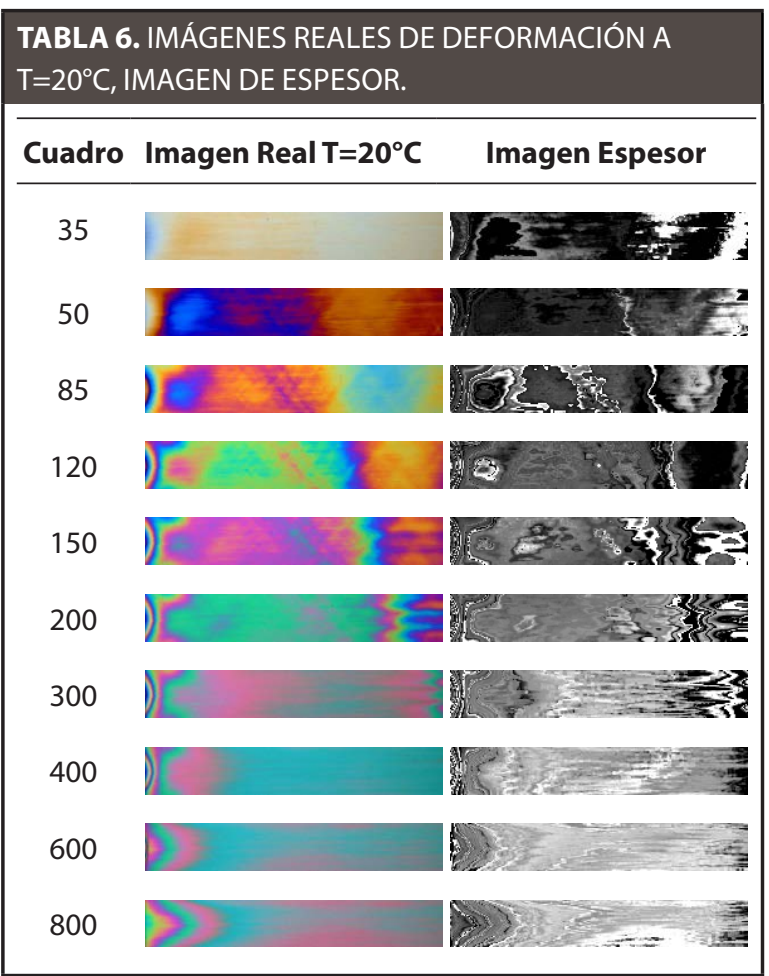

TABLA 7. IMÁGENES REALES DE DEFORMACIÓN A $\mathrm{T}=60^{\circ} \mathrm{C}$, IMAGEN DE ESPESOR.

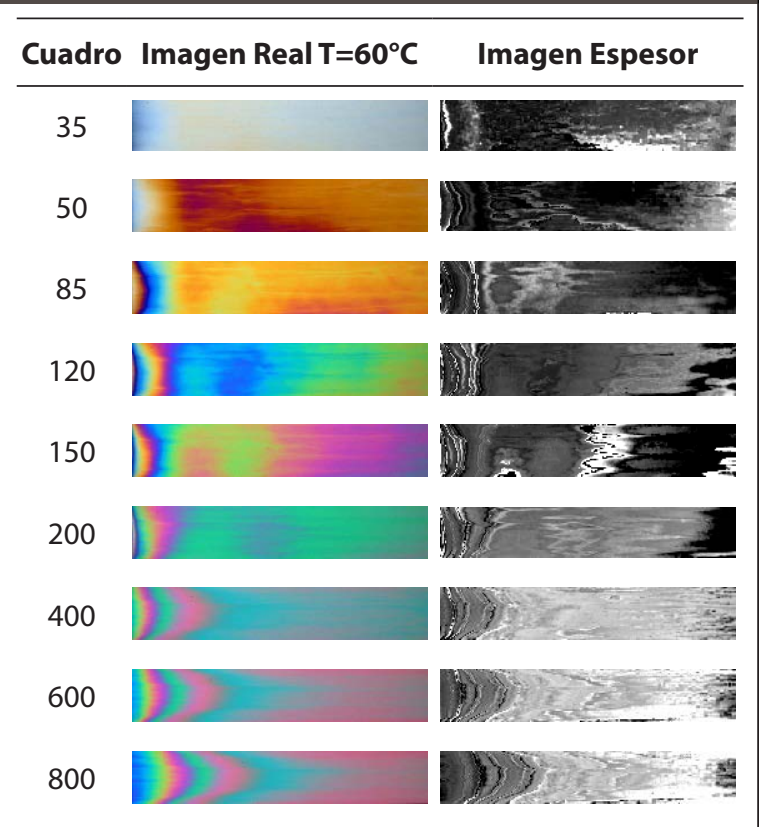

En las imágenes de espesor transformadas se observó regiones de mayores cambios de intensidad y que estos cambios están directamente relacionados con el cambio de esfuerzos y de espesor, por lo tanto con el fin de presentar el análisis, se muestra la evolución del espesor en un punto intermedio de la imagen (ver Figura 9) en la fila $90 \mathrm{y}$ columna 600 mostrado dicha evolución en la en la Figura 10 para la termodeformación a $\mathrm{T}=20^{\circ} \mathrm{C}$ y en la Figura 11 para $\mathrm{T}=60^{\circ} \mathrm{C}$.

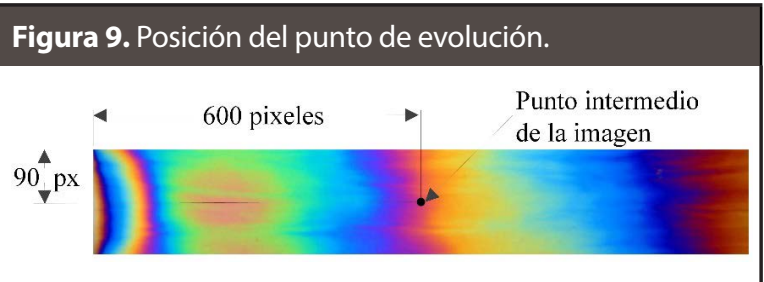

\section{Discusión}

En la Figura 10 se observa que en el cuadro 255 se tiene un espesor de 120 micras; en el cuadro 580 se alcanza 100 micras y finalmente en el cuadro 852 se reduce el espesor a 90 micras. De otro lado en la Figura 11 se encuentra que a 255 se alcanzó 100 micras y en el cuadro 400 se obtuvo 90 micras. Lo cual implica que la temperatura a $60^{\circ} \mathrm{C}$ permitió que los valores de espesor se redujeran más rápido con respecto al caso de deformación a temperatura ambiente, dado que a $20^{\circ} \mathrm{C}$ el material opuso mayor resistencia a la deformación. Por otro lado, sí observamos en la Figura $\mathbf{1 0}$ el valor de espesor para los cuadros 255 y 400 se puede ver que la diferencia son 5 micras, para el cuadro $400(13,3 \mathrm{~s})$ solo el espesor se redujo 20 micras $(3,7 \%)$ en la PPMC pero en la deformación a $60^{\circ} \mathrm{C}$ la reducción fue de 45 micras $(33,3 \%)$ en el cuadro 400.

En las imágenes de espesor obtenidas se puede observar que el espesor no es totalmente homogéneo debido a que la deformación se presenta por zonas en donde la película plástica opone menor resistencia. Cerca de la mordaza (lado izquierdo de la imagen) se presentan franjas con tonalidad blanca y otras negras, lo cual puede indicar que hay franjas 
en la película plástica durante la deformación que no presenta cambios de espesor.

En las curvas de evolución del espesor arrojan un resultado esperado ya que el espesor converge decrecientemente en el tiempo debido al estiramiento de la PPMC durante la deformación.

Se evidencia que hay alta fluctuación en pocos cuadros, lo cual puede explicarse por la falta de precisión del modelo ANFIS que evalúa la imagen, sin embargo, se deberán realizar trabajos futuros para explorar más las razones de dicha fluctuación.
El efecto de la temperatura sobre la deformación de la PPMC permite que se llegue a espesores menores en menor tiempo y a su vez la deformación sea de mayor homogeneidad.

\section{Conclusión}

La variación del espesor no es uniforme en la película plástica durante la deformación, pero se evidencia la reducción del espesor a medida que se desplaza el material sobre la región de interés, mostrado en la curva de evolución del espesor converge decrecientemente.

\section{Figura 10. Evolución del espesor en el pixel $(90,600)$. Tomada del video 9 con $\mathrm{T}=20^{\circ} \mathrm{C}$.}

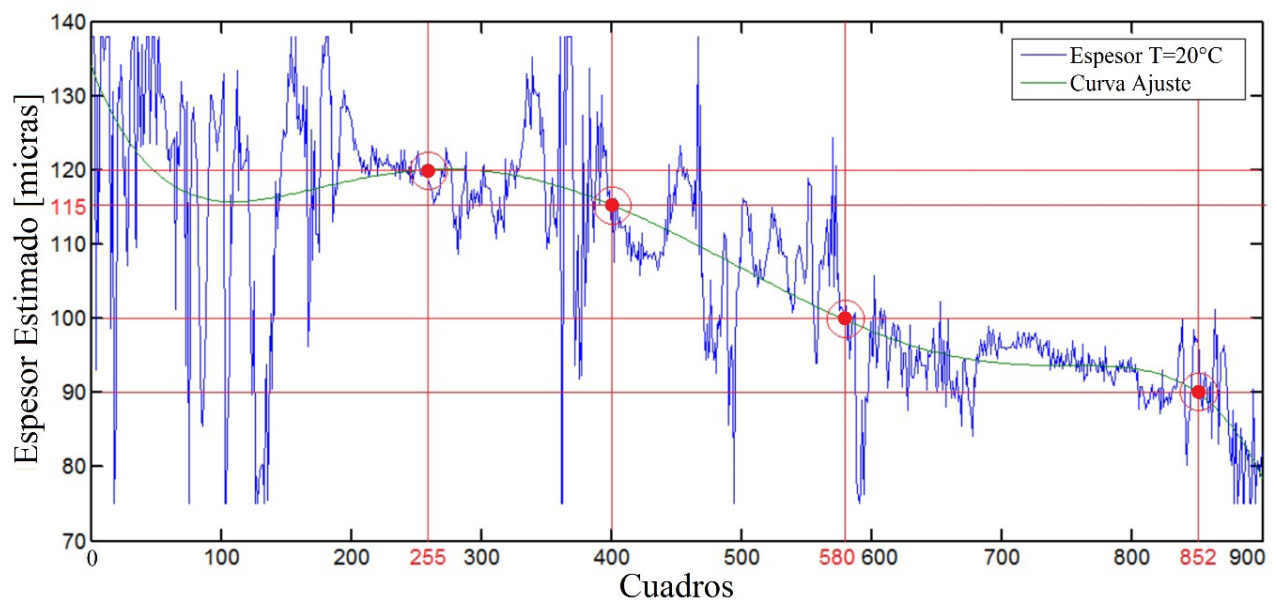

Figura 11. Evolución del espesor en el pixel $(90,600)$. Tomada del video 2 con $\mathrm{T}=60^{\circ} \mathrm{C}$

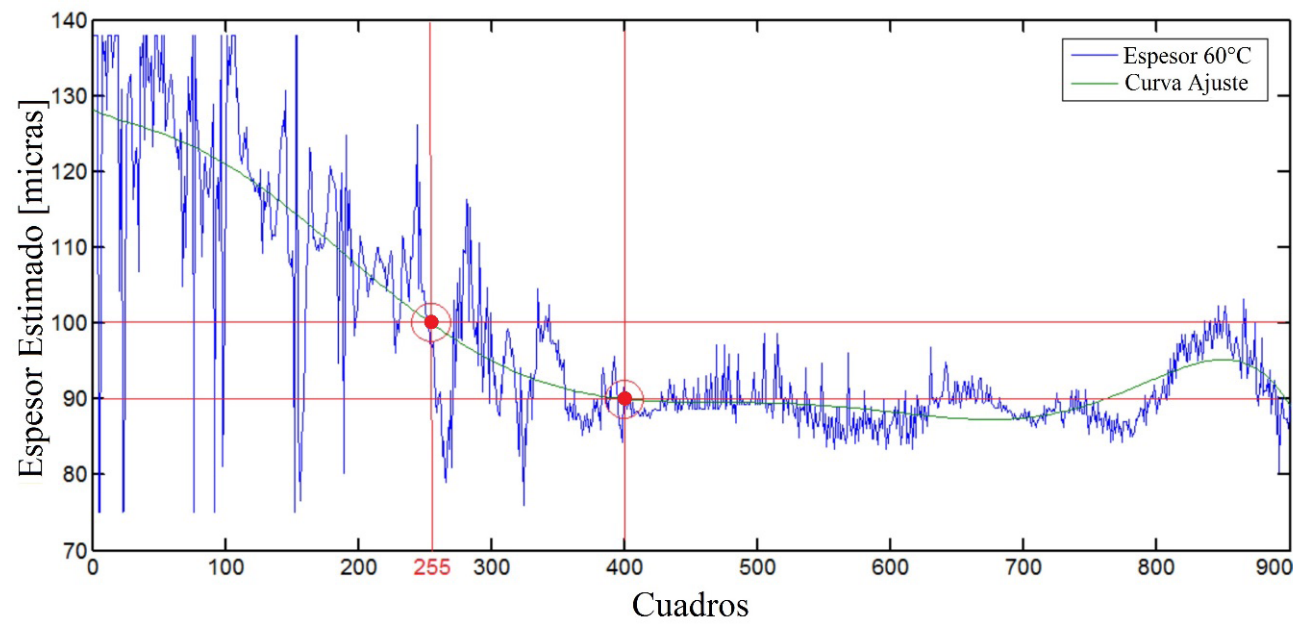


El uso del espacio de color HSI en el aprendizaje del espesor a través de la ANFIS mejoró los resultados ya que aumenta las características discriminantes del color.

Se logró describir la deformación de la PPMC usando una red ANFIS en donde se encontraron diferencias cualitativas analizando el comportamiento del espesor bajo efectos de la temperatura llevándonos a una conclusión por cada enfoque. La primera está en que los colores de interferencia si están directamente correlacionados con el espesor de la película, a través de una red Neuro-difusa se puede clasificar un color de interferencia dentro de un rango de espesor y que la temperatura favorece la homogeneidad de la deformación permitiendo así que los procesos de termoformado sean de mejor calidad.

\section{Referencias}

Agudelo, A. C. (2009). Evaluación y desempeño de láminas flexibles usadas en el empaque al vacío de productos carnicos. Tesis de Maestría en Ciencia y Tecnología de Alimentos. Universidad Nacional de ColombiaMedellín.

Ajovalasit, A., Petrucci, G., \& Scafidi, M. (2015). Review of RGB photoelasticity. Optics and Lasers in Engineering, 68, 58-73. https://doi.org/10.1016/j.optlaseng.2014.12.008

Araya, J., \& Cipriano, A. (2007). Optimal identification of Takagi-Sugeno fuzzy models for nonlinear FDI. Fault Detection, Supervision and Safety of Technical Processes 2006 (Vol. 1). IFAC. https://doi.org/10.1016/ B978-008044485-7/50128-7

Ashokan, K., \& Ramesh, K. (2009). Finite Element Simulation of Isoclinic and Isochromatic Phasemaps for Use in Digital Photoelasticity. Experimental Techniques, 33(1), 38-44. https://doi.org/10.1111/ j.1747-1567.2008.00368.x

ASTM D882-02. (2002). D 882: Standard Test Method for Tensile Properties of Thin Plastic Sheeting. Astm, 14, 1-10.

Briñez, J. C., Lopez, F., \& Restrepo, A. (2012). RGB Profiles in Digital Image Analysis for the Description of Interference Colors Produced on Photoelasticity Studies of the Plastic Films. In 2012 XVII Symposium of Image, Signal Processing, and Artificial Vision (STSI-
VA). Medellin.

Briñez, J. C., Restrepo, A., \& Branch, J. W. (2015). Evaluación Temporal de los Ordenes de Franjas de Color Utilizando Análisis de Saturación en Secuencias de Imágenes de Fotoelasticidad. In Décimo segundo Congreso Iberoamericano de Ingeniería Mecánica (CIBIM XII- 2015), Guayaquil-Ecuador.

Briñez, J. C., Restrepo, A., \& Branch, J. W. (2016). Timespace analysis in photoelasticity images using recurrent neural networks to detect zones with stress concentration. In Proc. SPIE 9971, Applications of Digital Image Processing XXXIX (Vol. 2, pp. 1-10). San Diego, California. https://doi. org/10.1117/12.2237373

Briñez, J. C., Restrepo, A., \& Lopez, F. (2013a). Evolución de la Intensidad de Pixeles para Describir la Deformación de Películas Plásticas. Tecnológicas, Medellín, 695-707.

Briñez, J. C., Restrepo, A., \& Lopez, F. (2013b). Métricas de similitud aplicadas para análisis de imágenes de fotoelasticidad. Dyna, Medellín, (1), 42-50.

Briñez, J. C., Restrepo, A., \& Lopez, F. (2013c). Morphological Analysis for Temporary Behaviour Description of Photoelasticity Images on Plastic Films. In L. N. in C. Science (Ed.), Huang F., Sugimoto A. (eds) Image and Video Technology - PSIVT 2013 Workshops. (pp. 122-132). Berlin, Heidelberg.

Dubelley, F., Planes, E., Bas, C., Yrieix, B., \& Flandin, L. (2016). Determination of the fracture energy in polymeric films by in situ photoelasticimetry on double edge notch specimen. Journal of Applied Polymer Science, 133(1). https://doi.org/10.1002/ app.42854

Editorial, G. (n.d.). Optical measurement techniques - A push for digitization.

Grewal, G. S., \& Dubey, V. N. (2007). Inverse problem of photoelastic fringe mapping using neural networks. Measurement Science \& Technology, 18(5), 1361-1366. https://doi.org/10.1088/09570233/18/5/024

Meléndez, F., Briñez, J. C., Restrepo, A., \& Branch, J. W. (2015). Poster: Identificación de variaciones del efecto de la temperatura en la deformación de películas plásticas analizando el comportamiento temporal de la fotoelasticidad. In XIV Encuentro Nacional De Óptica V Conferencia Andina y del Caribe En Óptica y sus Aplicaciones ENO - CANCOA 2015. Cali- 
Colombia (pp. 15-20). Cali: Universidad del Valle.

Mitra, S., \& Hayashi, Y. (2000). Neuro-fuzzy rule generation: survey in soft computing framework. IEEE Transactions on Neural Networks, 11(3), 748-768. https://doi.org/10.1109/72.846746

Navarro, H., Martínez, M., Saavedra, G., \& Pons, A. (2013). Photoelastic Analysis of Partially Occluded Objects With an Integral-Imaging Polariscope, (April 2014), 255-262.

Ramesh, K., \& Ramakrishnan, V. (2011). Digital photoelasticity - A comprehensive review. The Journal of Strain Analysis for Engineering Design, 46(4), 245266. https://doi.org/10.1177/0309324711401501

Solaguren-Beascoa Fernández, M. (2011). Data Acquisition Techniques in Photoelasticity. Experimental Techniques, 35(6), 71-79. https://doi.org/10.1111/ j.1747-1567.2010.00669.x

Swain, D., Philip, J., \& Pillai, S. A. (2014). A modified regularized scheme for isochromatic demodulation in RGB photoelasticity. Optics and Lasers in Engineering, 61, 39-51. https://doi.org/10.1016/j.optlaseng.2014.04.009

Swain, D., Thomas, B. P., Philip, J., \& Pillai, S. A. (2015). Novel calibration and color adaptation schemes in three-fringe RGB photoelasticity. Optics and Lasers in Engineering, 66, 320-329. https://doi. org/10.1016/j.optlaseng.2014.10.007
Lenio López Giraldo

PARA CITAR ESTE ARTÍCULO / TO REFERENCE THIS ARTICLE / PARA CITAR ESTE ARTIGO /

Meléndez Lozada, F.; Restrepo Martínez, A.; López Giraldo, F.E. (2018). Descripción del comportamiento de películas plásticas multicapa en procesos de termodeformación mediante análisis de fotoelasticidad digital. Revista EIA, 15(30), juliodiciembre, pp. 99-111. [Online]. Disponible en: https://doi. org/10.24050/reia.v15i30.1154 